\title{
Multi-Privacy Biometric Protection Scheme using Ensemble Systems
}

\author{
Marcelo Damasceno, A.M.P Canuto and Norman Poh
}

\begin{abstract}
Biometric systems use personal biological or behavioural traits that can uniquely characterise an individual but this uniqueness property also becomes its potential weakness when the template characterising a biometric trait is stolen or compromised. To this end, we consider two strategies to improving biometric template protection and performance, namely, (1) using multiple privacy schemes and (2) using multiple matching algorithms. While multiple privacy schemes can improve the security of a biometric system by protecting its template; using multiple matching algorithms or similarly, multiple biometric traits along with their respective matching algorithms, can improve the system performance due to reduced intra-class variability. The above two strategies lead to a novel, ensemble system that is derived from multiple privacy schemes. Our findings suggest that, under the worst-case scenario evaluation where the key or keys protecting the template are stolen, multi-privacy protection scheme can outperform a single protection scheme as well as the baseline biometric system without template protection.
\end{abstract}

\section{INTRODUCTION}

Biometrics is an enabling technology that uses personal biological attributes such as face, fingerprint, iris, and signature, that can uniquely characterise an individual. However, this uniqueness property also becomes its potential weakness when the template of the system is stolen or compromised. Even though a biometric template may contain only some extracted features, for some biometric traits like fingerprint, it is possible to approximately reconstruct a digital copy of the biometric trait [1]. Therefore, biometric-based systems need to consider its template security by providing methods to revoke templates when they are compromised [2]. To this end, we consider a number of strategies to improve biometric template protection in this study, namely, (1) using multiple privacy schemes and (2) using multiple matching algorithms. While multiple privacy schemes can improve the security of a biometric system by protecting its template, using multiple matching algorithms or similarly, multiple biometric traits along with their respective matching algorithms, can improve the system performance due to reduced intra-class variability [3]. The above two strategies lead to a novel, ensemble system that is derived from multiple privacy schemes.

In a biometric system enhanced with a privacy scheme, a stolen template cannot be introduced into another system

Marcelo Damasceno is a lecturer in Federal Institute of Rio Grande do Norte and $\mathrm{PhD}$ student in the Department of Informatics and Applied Mathematics in the Federal University of Rio Grande do Norte, Brazil who A.M.P Canuto is his supervisor (email: marcelo.damasceno@ifrn.edu.br, anne@dimap.ufrn.br).Norman Poh joined the Department of Computing as a Lecturer. He received the Ph.D. degree in computer science in 2006 from the Swiss Federal Institute of Technology Lausanne (EPFL), Switzerland (n.poh@surrey.ac.uk). This work was supported by CNPq, Conselho Nacional de Desenvolvimento Científico e Tecnológico - Brazil or be directly associated with the user. Each biometric template protected by a privacy scheme is unique only for use by the intended biometric system. An important class of privacy scheme deployed for biometric systems is known as "cancellable biometrics" which transforms or intentionally distorts the original biometric samples to protect the user's privacy. Therefore, the enhanced biometric system compares two samples - the query sample that is collected live and the template that has been collected during enrolment in the distorted feature space. Unfortunately, the use of distorted biometric samples usually not only decreases the system performance due to the introduced distortion function or noise but also increases the computational complexity in the matching process. Therefore, it is important for a privacy protected biometric system to support a good tradeoff between discrimination capability as well as the degree of non-invertibility of the distortion function in exchange for a higher level of security.

Although a number of methods exist to combine information sources, as described in [4], few studies systematically explore the effectiveness of multiple cancellable functions or protection schemes in the context of information fusion. The literature on information fusion considers the following classes of systems: (i) multimodal system, which combines multiple biometric traits collected by different sensors; (ii) multi-algorithmic system, which uses different features of the same biometric modality; (iii) multi-sample system, which combines several samples or instances of the same biometric modality; (iv) multi-sensor system, which recognises a user based on one biometric modality through the combination of different sensors (of the same modality). A special case of multi-algorithmic biometric system is to use several biometric feature representations, leading to a multi-feature biometric system. Although recent progress on biometric information fusion includes the combination of auxiliary, non-discriminatory information such as userspecific characteristics and biometric sample quality [5], as well as combining multiple biometrics in the context of template protection [6], the property of using multiple protection schemes is not often not systematically evaluated, at least not in the context of information fusion.

In addition, nearly all the studies on multibiometric template protection focus on physiological biometrics such as face, fingerprint, and iris, e.g., [cite works 40-44, 6 of Rathgeb's paper]. The level of protection for behavioural biometrics, where the sample has much more variability, and arguably less unique to an individual, and the possibility of change in behaviour over time, merits an investgation on its 
own right.

The closest work on multiple protection schemes applied to the behaviour biometrics is [7], which suggests that an ensemble system that is generated by one cancellable function has similar (Interpolation and Double Sum functions) statistical performance compared with performance achieved by the baseline ensemble system without any cancellable functions. In addition, the authors showed that the use of BioConvolving to the ensemble method can significantly further improve the statistical performance of the ensemble compared with the baseline system. We extend this work to include BioHashing. Apart from adding more cancellable functions, we also systematically evaluate the performance of the ensemble using all possible combinations of the constituent cancellable functions. Moreover, we compare each of the possible combinations of ensemble with their respective baseline systems (without the cancellable function) as well as with a single privacy protection scheme.

Although the multi-privacy ensemble system improves the recognition accuracy and the security of the overall system, there are other issues not falling into the scope of the study. We do not address the issue of increased computational complexity as any improved security is expected to trade off against the ease of use of the system. Another more important issue is related to usability: how a biometric user can use and properly manage the different keys needed by the cancellable functions. This problem is not particularly crucial for cancellable biometrics because even if an impostor possesses the key used by a cancellable function, he/she cannot recover the original biometric sample because the cancellable functions are non-reversible. How to practically help the user to manage or store the keys is a usability issue that merits a proper study on usability as well as key design and management.

In summary, the contributions of this paper are four-fold: (1) proposal of a multi-privacy protection scheme applied to biometrics; (2) performance evaluation of multi-biometric system using sub-sets of protected biometric traits; (3) understanding the system performance in the context of decision fusion, based on the ensemble method; and (4) applying the system to behavioural biometrics.

This paper is divided into eight sections and it is organized as follows. Section I showed the motivation and paper contributions, Section II describes the subject background of this paper, while the ensemble systems are described in Section III. The cancellable transformations are described in Section IV and the behavioural biometric dataset TouchAnalytics is discussed in Section V. The Experimental Methodology is explained in Section VI, while the Section VII presents and discusses the results obtained in the experiments. Finally, Section VIII presents the final remarks of this paper.

\section{BACKGROUND}

\section{A. Related Work}

Multi-biometric template protection systems offer significant advantages, improving public confidence and acceptance of biometrics because it can balance trade-off between accuracy and security. Thus, this subsection focuses four papers closely related with our proposal.

The paper wrote by Rathgeb and Busch [6] presents a discussion about issues and challenges of multi-biometric template protection. The authors summarise and discuss some characteristics of multi-biometric template protection papers, which are: (1) fusion of biometric traits in feature level, score level and decision level; (2) rearrangement of biometric representation to provide a uniform distribution of error probabilities; (3) combination of biometric modalities increases security and accuracy; (4) use of different feature extraction methods using one biometric modality; (5) use of multi-biometric protection system using multiple protected physical modalities. However, Rathgeb and Busch [6] do not discuss the use of behavioural biometric in multi-biometric protection systems. Therefore, this paper fills this gap investigating the use of multi-biometric template protection systems using behavioural modalities.

Canuto et al. [8] investigates the performance of system using multiple physical biometric samples. However, this paper investigate the use of multiple behavioural biometric samples. The use of behavioural biometrics is more challenging than the use of physical because human behaviour is influenced by different factors [9].

The empirical experiments performed in [9] show the use of a single biometric samples multi-algorithm approach, archiving interesting results in protected and unprotected biometric samples. Unfortunately, the authors do not show the advantageous of using multiple samples. This paper, consequently, extends our previous work [7], adding a multiple biometric samples approach in a multi-algorithm biometric system.

Kelkboom et al. [10] discusses the application of template protection in a multi-algorithm approach using a 3D-face modality. The authors discuss the findings using different fusion levels, feature-, score- and decision-level. Instead of use of a majority voting (our proposal), the authors applied the rules AND and OR to fusion the decision of biometric system.

\section{B. Cancellable Biometrics}

Biometric sample is user-specific since the biometric characteristics is unique and it is hard to be replaced in case of being stolen or lost. One of the main problems of security faced by biometric system is an unauthorized copy of the stored biometric template [8]. Hence, biometric templates must be stored in a protected way using a protection scheme that possesses the following four properties [2]: (1) Diversity: the secure template must not allow crossmatching across databases; (2) Revocability: it should be easy to revoke a compromised template; (3) Security: it must be computationally hard to obtain the original biometric template from the secure template; and (4) Performance: the biometric template protection scheme should not degrade the recognition performance of a biometric system. 
Unfortunately it is difficult to define a template protection that can satisfy all these characteristics due to the tradeoff among them. In this way, several template protection methods have been proposed in the literature [8]. In [2], these methods were broadly divided in two approaches, which are: (1) Biometric cryptosystem: Some public information about the biometric template is stored and it is usually referred to as helper data. From a cryptography system's perspective, biometric cryptosystems are also known as helper databased methods. (2) cancellable transformation: In this case, a transformation function $(f)$ is applied to the biometric template $(T)$ and only the transformed template $(f(T))$ is stored in the database. The original template $T$ is hard to be obtained from $f(T)$.

Unfortunately, to compute $f(T)$ is required a key $k$. The use of different keys generates inconvenience to users due to necessity to present a key to each biometric sample used in a biometric system. In our solution, each cancellable function $f(x)$ requires its own key $k$. Thus, a user will need to present $n$ different keys $k_{i}, i \in\{1, \ldots, n\}$ to each cancellable function. Our solution is not ideal, but we are working in a new way to use cancellable template without any or several keys.

\section{ENSEMBLE SYSTEMS}

Ensemble systems, also known as multi-classifier systems or fusion of experts, exploit the idea that different classifiers can offer complementary information about a sample that is being classified; thereby improving the overall recognition performance [11]. The improvement can be explained by the diversity of the constituent classifiers in terms of performance, as well as their underlying dependencies. Indeed, it is possible to fully predict the fusion system given the multidimensional class-conditional score distributions (for the genuine and impostor classes) are known under a linear fusion operator [3].

There are two main issues to consider in the design of an ensemble: the ensemble components and the combination method. In relation to the first issue, the ideal situation would be to choose a set of base classifiers with uncorrelated errors, which would be combined in such a way as to minimize the effect of these failures. Depending on its particular structure, an ensemble can be realised using two main approaches: heterogeneous, i.e, to combine different types of classification algorithms; and homogeneous, i.e., to combine classification algorithms of the same type.

The next step is to choose an effective way to combine the components outputs, which is usually a typical decision-level fusion method. If there are $I$ component classifiers, there will be $I$-choose- $n$ possible ways of choosing the ensemble decision. The combination of the classifiers outputs diverge from the simplest approach using class labels or rank values to the utilization of more elaborate information, such as support degree $D$ [11].

The use of different component classifiers in a ensemble is a standard way to promote diversity. The common combination ensemble methods such as bagging, boosting, stacking, and voting [11] promote classifier diversity. This paper uses the majority voting approach, which is based on democracy voting system. The majority output class label of the classifiers is the final output, i.e, each classifier presents its class label output and the majority class label is the winner. Voting (majority vote) decision fusion was selected because it is a simple combination method which combines homogeneous and heterogeneous components decision. Our paper uses the voting in a user identity level, i.e, each component votes in what type of user the claimed individual is. The decision of ensemble is the majority voted user (client or impostor).

Ensemble systems can use cancellable biometrics and achieves very promising results [8, 12]. These papers show the accuracy of biometric system using cancellable biometric sample can be improved using an ensemble system. However, the authors use only physical biometric modalities. Unlike these studies, this paper applies a multi-privacy scheme using ensemble systems using behavioural biometrics for user verification task.

\section{Cancellable Functions}

Cancellable transformation functions transform the biometric samples in such a way that it is computationally hard to get the original form [2]. Unfortunately, high variance and noise are unintended consequence of transforming a biometric sample using cancellable functions. Consequently, user verification becomes more difficult. Thus, verification and authentication systems which use cancellable biometric samples must provide better performance than systems using non-modified data.

The cancellable functions can be further classified as salting and non-invertible transformations. In the first case, the transformation function $f$ is invertible, while $f$, as implied, is either non-invertible or hard to invert in the second case. The use of a one-way function $f$, easy to compute (in polynomial time) but hard to invert (given $f(x)$, the probability of finding $x$ in polynomial time is small), is the main purpose of using non-invertible transformations. Hereafter, the terms transformation function or cancellable transformation will be taken as referring to the non-invertible case.

The literature reports that ensemble systems offer better performance than single classifiers [11] and performs well in some cancellable biometric datasets [9]. Thus, we analyse ensemble systems applied to behavioural biometric samples using the following cancellable functions: Interpolation, BioHashing, BioConvolving and Double Sum. All the transformation functions are better described in [8].

\section{A. Interpolation}

This technique is based on polynomial interpolation. It consists of generating a new biometric model by extracting function points resulting from the attribute interpolation process in such a way that the original attributes are preserved. The attributes compose the original biometric model. 
Although it is simple, this algorithm makes the inversion of the transformed function difficult, thus generating a reasonable level of security to the system. Therefore, it is very efficient in satisfying two of the main requirements for transformation techniques, which are simplicity and efficiency at the same time.

\section{B. BioHashing}

BioHashing technique has originally used in other biometric modalities, such as fingerprint, palm and face [8]. BioHashing algorithm is characterized by transforming original biometric into a non-invertible binary sequence. This invertible binary sequence is based in a inner product between the original biometric vector and each pseudo-random orthonormal vector $o_{i} \in \mathbb{R}^{n} \mid i=1, \ldots, m$. Each $o_{i}$ is obtained using the Gram-Schmidt algorithm with original biometric data as input.

BioHashing technique has originally used in other biometric modalities, such as fingerprint, palm and face [13]. In this work we use the original BioHashing algorithm but in future we will use the adaptation developed by our group, described in [8].

\section{BioConvolving}

BioConvolving method was originally proposed for signature [8]. In this method, the transformed functions are created through linear combinations of sub-parts of the original biometric template $\Gamma$. Basically, this method divides each original biometric sequences into $W$ non-overlapping segments, according to a randomly selected transformation key $d$. Then, the transformed functions are obtained by performing a linear convolution between the obtained segments.

According to [14], the BioConvolving security approach is based on the fact that a blind deconvolution problem is needed in order to retrieve the original template. Moreover, in [14] it was also shown for signature that even if multiple transformed templates are stolen, it is not possible to retrieve the original template.

\section{Double Sum}

Double Sum cancellable transformation is a simple method and it consists of summing the attributes of original biometric model with two other attributes of the same sample. In other words, each attribute of original biometric model is transformed into the sum of three attributes randomly chosen. In this case, even if an impostor has access to transformed samples, it will not be possible to define original samples from transformed one.

The double sum method can be considered as noninvertible, since the number of possible combinations is very high. For example, if a biometric model has $n$ attributes, there will be

$$
C_{s}=n !^{3}
$$

possibilities of generating the encrypted content. Therefore, it is computationally infeasible to reverse the process in a feasible time, especially for large $n$ that is greater than
50 ; thus, justifying the non-invertibility and security of the scheme.

The revocability of this method is guaranteed by the security key. Indeed, the key is responsible for the reorganization of the original biometric sample and for the choice of the original attributes that will be summed. In case of being lost or stolen, a new transformation model can be created using a different security key $k$. In this paper, we are using the same security key $k$ and the dimension of the transformed model is the same of the original dataset.

\section{DATASET}

The TouchAnalytics [15] dataset is a behavioural trait collected through strokes in mobile touchscreen interaction. TouchAnalytics is composed of 30 attributes derived from observed strokes performed by 41 users. The number of stroke attempts is different for each user. Thus, a stroke is defined in [15] as a trajectory encoded as a sequence of vectors with real numbers, $s_{n}=\left(x_{n}, y_{n}, t_{n}, p_{n}, A_{n}, o_{n}^{f}, o_{n}^{p h}\right)$ where $n \in 1,2, \ldots, N$ enumerates the number of strokes (and there are $N$ strokes), with location $x_{n}, y_{n}$, time stamp $t_{n}$, pressure on screen $p_{n}$, area $A_{n}$ occluded by the finger, finger orientation $o_{n}^{f}$, and phone orientation $o_{n}^{p h}$ which can be landscape or portrait. Therefore, it is possible to derive the information about area covered, stroke pressure, direction, velocity and acceleration from the above raw data.

TouchAnalytics is divided in horizontal and scrolling (vertical) movements. Thus, it is possible to analyse the user's orientation patterns.

Frank et al. [15] presented some initial results using three different scenarios, namely: (1) Inter Session: The goal is to authenticate users across multiple sessions performed in the same day; (2) Inter Week: The goal is to authenticate users in two different sessions (the period of time between these two sessions is one week); (3) Intra Session: All the user data was used in the process, time independently.

In [15], the authors presented some results using $k$-NN and SVM (Support Vector Machine) classifiers. In addition, the authors use Equal Error Rate (EER) metric which reports the performance of the system when the probabilities of false acceptance and false rejection are equal.

According to Frank et al. [15], the median EER achieved ranges from $0 \%$ to $4 \%$ across all sessions: Inter Session, Inter Week and Intra Session. The median EER in Intra Session are $0 \%$. It seems that, within one session, most users do not considerably change their touch behaviour. Inter Session EER reaches from $2 \%$ to $3 \%$ and Inter Week EER reaches from $0 \%$ to $4 \%$. These results indicate that behavioural biometrics (touch data) have good perspectives in practical use.

\section{Methodology}

This section describes the the proposed multi-privacy protection scheme. In this scheme, each target user has his/her own dedicated classifier. In order to train this userspecific classifier, the construct a training data set consisting of positive stroke samples, i.e., samples belonging to the target user (or client/match samples) and negative stroke 
samples, i.e., samples belonging to the remaining users (or impostor/nonmatch samples). If there are $J$ users, than, the user-specific training set consists of one part of positive training data and $J-1$ parts of negative training data. In order to adjust for the unbalanced user-specific training data sets, we resample the negative stroke samples such that every sample as a probability of $\frac{N_{p}}{N_{n c}}$ of being selected, where $N_{p}$ is the number of positive samples and $N_{n c}$ is the number of negative samples. Therefore, by using the above resampling method, each user-specific classifier is trained with $N_{p}$ positive samples as well as $N_{p}$ negative samples, thus creating a balanced training set.

Thus, our balanced user-specific dataset has $N_{p}$ positive instances and $N_{n c} \times T$ negatives instances.

In the next step, we apply four cancellable transformation functions to create four corresponding protected balanced user-specific datasets. The Figure 1 illustrates these 2 steps: (1) splitting and balancing the main dataset by user and (2) applying the four cancellable functions. The next step is to model the decision module after cancellable biometric samples have been generated.

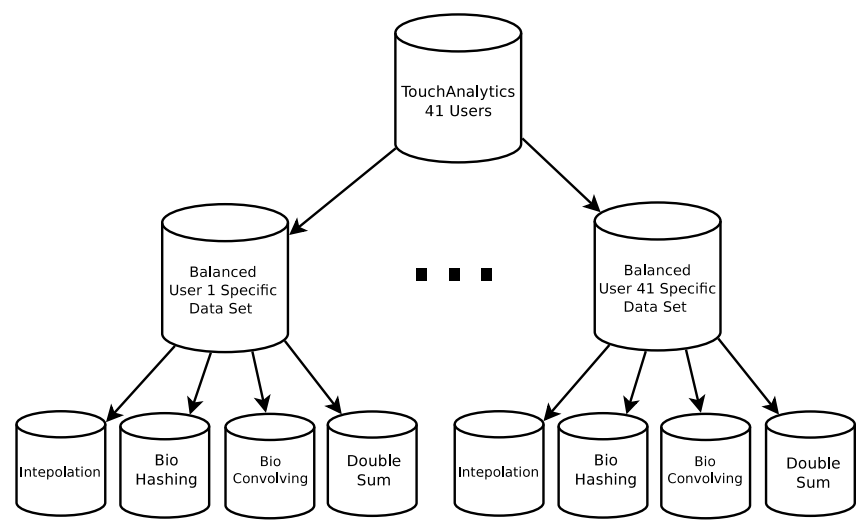

Fig. 1. Generation of cancellable biometric samples for each user

The system architecture is composed of three components: (1) a pool of cancellable biometric schemes, (2) an ensemble system, and (3) a decision fusion which is based on the majority vote. If there are $N$ cancellable schemes, then, there are at most $\sum_{k} C_{k}^{N}$, for $k \in\{2, \ldots, N\}$ possible ways of constructing the ensembles. For example, in our case, there are 11 possible ensemble systems derived from four cancellable schemes $(N=4)$, we will consider three scenarios, each with a different number of cancellable schemes, namely: (1) the scenario with two cancellable schemes: $C_{2}^{4}=$ $4 \times 3 / 2=6$ combinations; (2) three scheme scenario: $C_{3}^{4}=4$ combinations; and finally (3) the scenario in which all the four schemes are used: $C_{4}^{4}=1$ combination. Figure 2 shows the architecture of the system along with the decision module which is based on the voting scheme.

Each of the 11 combinations is referred to by the conjunction of the first four letters of each constituent cancellable scheme, namely, Inte for Interpolation, BioH for BioHashing, BioC for BioConvolving, and Doub for DoubleSum. For example, the scenario 'InteDoub' refers to the ensemble consisting of the Interpolation and the Double Sum schemes.

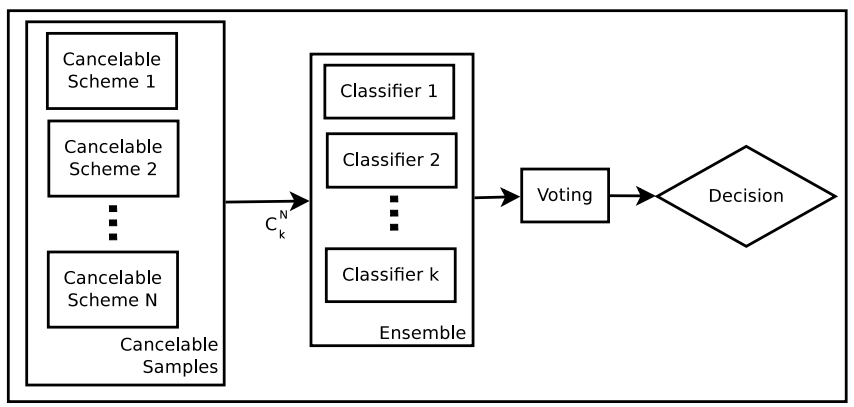

Fig. 2. Flowchart represents the system architecture

In addition, for simplicity, we limit ourselves to using only majority vote to combine the decision of $k$ classifiers.

The ensemble system is composed of classifiers $k-\mathrm{NN}$, SVM, Naive Bayes and MultiLayer Perceptron. These classifiers were selected because they provide diverse learning approaches and error bias. These characteristics increase the ensemble diversity, which usually means leads to an improvement in performance.

Our empirical study evaluates its performance using a modified 10-fold cross-validation method. Our modified 10fold cross-validation ensures that the training set does not have any biometric sample of subjects who are present in the test set. Therefore, we test our system with biometric samples that have not been processed in training phase. All results presented in this paper refer to the mean over all balanced user-specific datasets using 10-fold cross-validation technique.

The classification algorithms of this investigation were extracted from WEKA $^{1}$ package. In addition, an exhaustive investigation using a cross validation approach defined the values of supervised learning algorithms parameters. Consequently, the algorithms were executed with the following parameters: $k$-NN with $\mathrm{k}=5$; SVM with polynomial kernel (grid-search), pruned decision tree, MLP (one hidden layer) and Naive-Bayes with standard WEKA settings. The next section will present and discuss the relative change of EER values by combination experiment.

\section{RESUlts AND Discussion}

This section presents two different analysis: (1) comparison of multi-privacy biometric scheme vs. single privacy biometric scheme and (2) the relative change of $\operatorname{EER}(\%)$ in multi-privacy scenarios compared with baseline dataset (Original dataset). Original dataset is a set of non-modified biometric samples.

We use the Voting results available in [7] to compare the results of multi-privacy against the single privacy biometric scheme. The Table I presents the mean EER and standard deviation of voting decision fusion using only one protected biometric sample [7].

\footnotetext{
${ }^{1}$ http:www.cs.waikato.ac.nz/ml/WEKA
} 
TABLE I

Voting - Eer - Percentage. Source: [7]

\begin{tabular}{r|l|l|l|l} 
& \multicolumn{2}{|c|}{ Horizontal } & \multicolumn{2}{c}{ Scrooling } \\
\hline scenario & Median & $\begin{array}{l}\text { Standard } \\
\text { Deviation }\end{array}$ & Median & $\begin{array}{l}\text { Standard } \\
\text { Deviation }\end{array}$ \\
\hline Original & 9.7 & 5.8 & 8.9 & 6.4 \\
\hline Interpolation & 13.7 & 5.8 & 10.9 & 6.7 \\
\hline BioHashing & 33.5 & 9.7 & 32.6 & 12.6 \\
\hline BioConvolving & 0.2 & 0.4 & 3.6 & 11 \\
\hline DoubleSum & 11.9 & 6.9 & 11.4 & 7.5 \\
\hline
\end{tabular}

Figures 3 and 4 exhibit the boxplots of EER by Scenario achieved in this paper, using horizontal and scrolling strokes respectively. The boxplots represent the EER median values organized in an ascendind order, for better comparison and readability

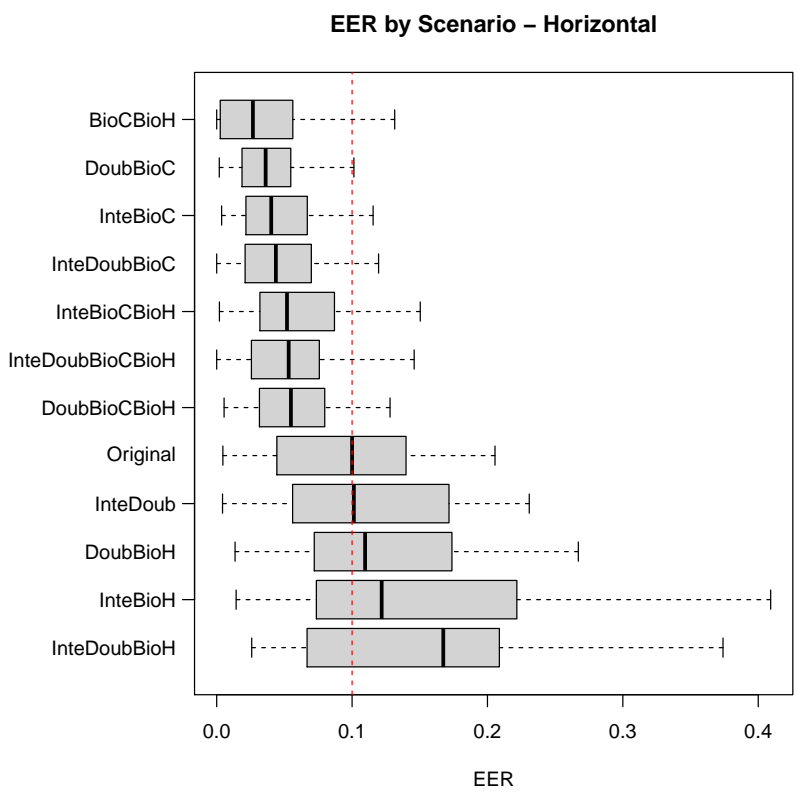

Fig. 3. BoxPlot of horizontal scenarios

To analyse the multi-privacy scheme vs. single-privacy we will check if multi-privacy can outperform the second and third best median EER (Double Sum and Interpolation) present in Table I. We don't analyse the better case (BioConvolving) because its result in single protected scenario is very good and hard to improve, $0.2 \%$ and $3.6 \%$ using horizontal and scrolling strokes, respectively.

Using horizontal strokes, multi-privacy methods using Interpolation and Double Sum samples increase the performance of Interpolation, Double Sum single-privacy scenarios in five out of seven cases (71.42\%). Four cases are better $(57.14 \%)$ using scrolling strokes.

The performance of multi-privacy protection scheme using BioHashing dataset together with at least one protected sample increases in $100 \%$ of cases, when compared with single privacy scheme using BioHashing samples. See BioHashing results presented in Table I and compare with BioHashing

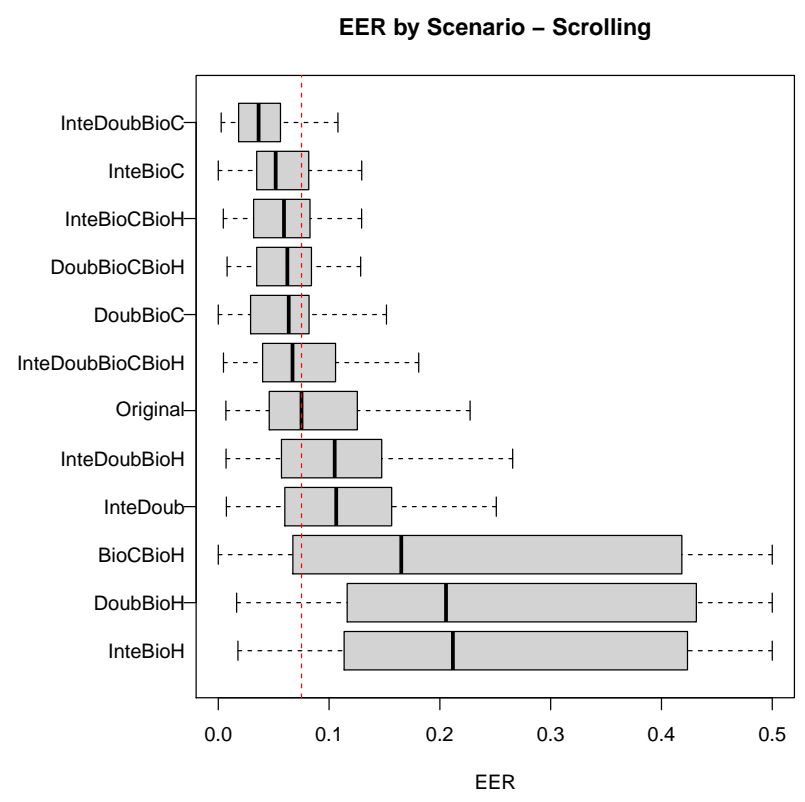

Fig. 4. BoxPlot of scrolling scenarios

boxplots present in Figures 3 and 4. This is an important finding because the use of a cancellable biometric trait with poor performance (BioHashing) can increase the performance of a biometric system, when it is combined with a different protected sample.

In both scenarios (horizontal and scrolling strokes), the multi-privacy scenarios with BioConvolving achieve lower median EER than median of single-privacy scenarios, i.e, the use of BioConvolving samples increases the performance of multi-privacy scheme compared with single-privacy schemes. We can observe that all multi-privacy scenario boxplots using BioConvolving have median values (Figures 3 and 4) under all median values of single scenarios (Table I) (except BioConvolving).

When comparing both directions, as in [9], the results using horizontal strokes were better than scrolling ones. Using as metric the accumulated EER, we have a accumulated EER of 90.9 and 140.6 for horizontal and scrolling datasets respectively. We can observe a difference between horizontal and scrolling accumulated EER of 49.7 .

The EER of the scenarios using all protected biometric samples is among TOP 5 results (Horizontal $=5$ th, Scrooling $=3$ rd), both lower than Original dataset. This result shows that the use of all protected samples increase the performance of the biometric system compared to its use with original data, In addition, it minimizes all the disadvantages related to use of a single non-protected scheme.

In our second phase of investigation, we will analyse the different multi-privacy scenarios taking account the relative change of $\operatorname{EER}(\%)$ using the multi-privacy scenarios and Original samples (non-modified biometric sample). The Figures 5 and 6 exhibit the boxplots ploted with relative change of $\operatorname{EER}(\%)$ using horizontal and scrolling strokes, 
respectively. As Figures 3 and 4, the Figures 5 and 6 are organized in a median ascending order.

The relative change of combination scenarios is obtained by:

$$
\frac{E E R_{\text {CombinedScenario }}-E E R_{\text {Original }}}{E E R_{\text {Original }}} \times 100 \%
$$

Relative Change EER(\%) by Scenario - Horizontal

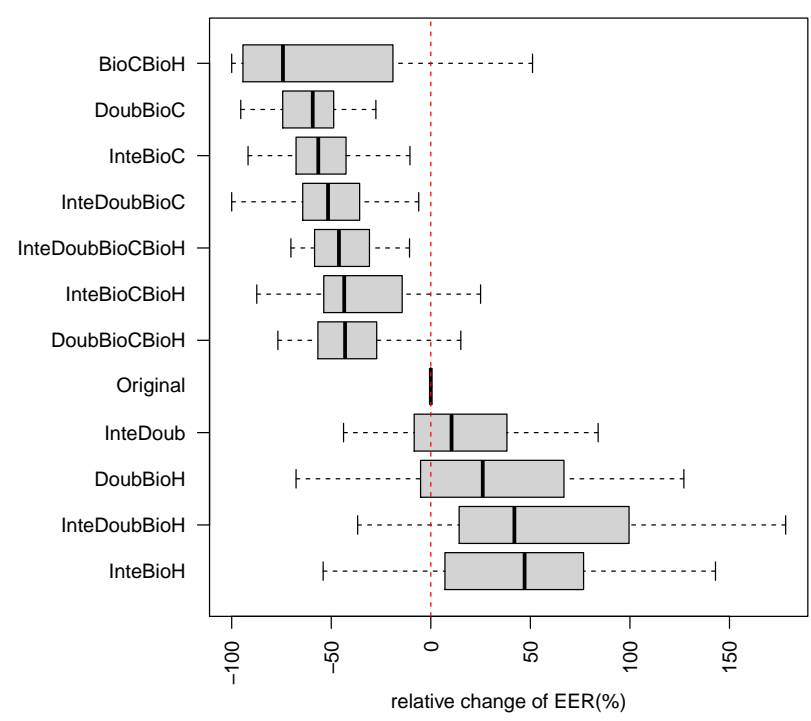

Fig. 5. BoxPlot of relative change of $\operatorname{EER}(\%)$ using horizontal scenarios

Relative Change EER(\%) by Scenario - Scrolling

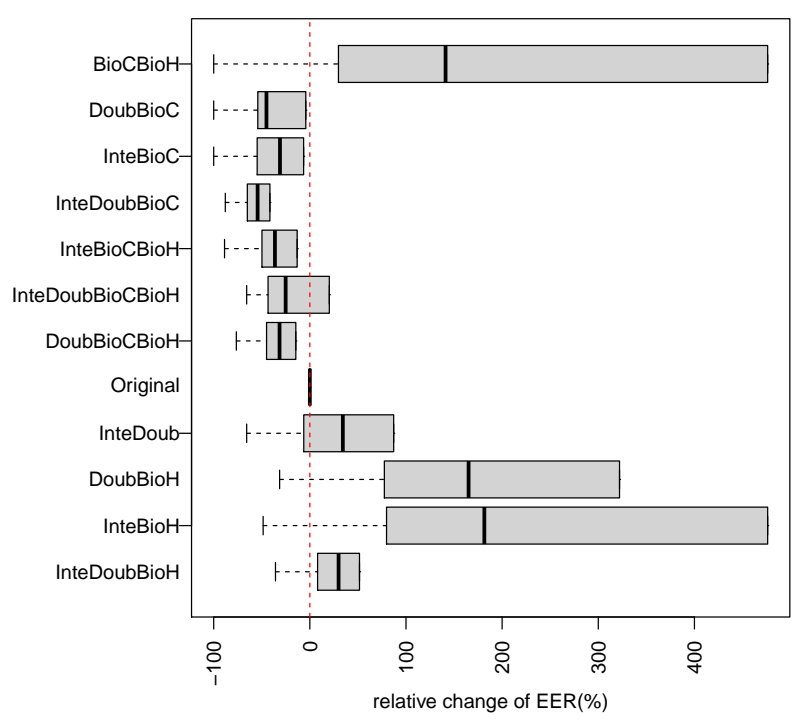

Fig. 6. BoxPlot of relative change of EER(\%) using scrolling scenarios

The boxplots presented in Figure 5 show that 7 scenarios $(63.63 \%)$, out of 11 , are better than Original dataset results.
In addition, the use of another privacy method together with BioHashing decreases significantly the EER of BioHashing samples, 28.6\% in the best case (BioHashing EER BioCBioH EER).

The boxplot present in Figure 6 show that 6 scenarios schemes, out of $11(66 \%)$, are better than Original dataset scenario. The same thing happened to BioHashing using scrolling strokes. The EER decreased $24.9 \%$ in the best case (InteBioCBioH) using BioHashing with another protected sample.

These findings show that we can use multi-privacy protection scheme using voting decision function without deteriorating the performance of the biometric-based authentication system, compared with the use of non-modified biometric sample. As well, in most cases, using only one protected biometric sample. The multi-privacy protected scenarios performs better in at least $54,4 \%$ of cases, when compared with Original sample use.

\section{FINAL REMARKS}

In this paper, we propose and evaluate a multi-privacy biometric protection scheme using ensemble systems. The multi-privacy scheme combines the use of multi-algorithm and multiple protected biometric samples. Four cancellable transformations (Interpolation, BioHashing, BioConvolving and Double Sum) were used to protect a behavioral modality (TouchAnalytics). The multi-privacy protection scheme was applied to analyse the performance and security of a biometric system using ensemble systems and cancellable biometric samples.

Multi-privacy protection scheme achieved better performance compared with single protection scheme using Original samples. Using horizontal strokes we obtained 7 (of 11 scenarios) better performance scenarios and 6 (of 11 scenarios) better scenarios using scrolling strokes, both compared with the Original dataset scenarios.

Multi-privacy protection scheme has better performance than single protection scheme, both using a complex protected biometric sample. A combination of a complex and a non-complex protected biometric sample in a multi-privacy scheme increases the performance compared with the single scheme using the same complex sample. In our case, the use of another privacy method together BioHashing decreases significantly the EER of Biohashing, $28.6 \%$ in the best case $(\mathrm{BioCBioH})$. Using both stroke directions, the performance (EER) of multi-privacy scheme using BioHashing droped by $28.6 \%$ (BioCBioH - Horizontal) and $24.9 \%$ (InteBioCBioH - Scrolling) in the best cases.

Multi- privacy protection scheme increase the performance and security of biometric systems. Using the relative change of EER (\%) of multi-protection scheme, the scenario using all the protected samples is in 5th and 6 th place using horizontal and scrolling data, respectively. This result shows that multi-protection scheme increases the performance of a biometric system compared with its use with the original biometric samples. Beyond this, the use of multiple protected biometric samples provides more security 
because the success rate of attacks such as shoulder surfing, forgeries or brute force decreases.

Multi-privacy scheme improves the use of safe biometric authentication systems. The use of diverse cancellable biometric samples requires different keys to code the biometric sample. Thus, users can present only part of encoding keys to the multi-privacy scheme because this scheme can process a subset of protected representation. From our results, we see that use of only two different protected data presents low EER values for both stroke directions.

Proposal Limitation Multi-Privacy Protection Scheme (MPPS) increases user inconvenience because the individual needs to present more than one key to encode his biometric sample during user verification. In addition, the processing time of multi-privacy scheme is higher than use of only nonprotected biometric sample but the increase of perfomance and security can overcome this limitation. There is no guarantee here that MPPS, which is a specialized ensemble method, can be better than any protected biometric sample. In our case, any multi-privacy scenarios were better than BioConvolving (Table I), Figures 3 and 4).

Scenarios Limitation Our scenarios just model the solution based on protected biometric representation. Hence, we need to taking into account user-specific samples because user samples influences the biometric system performance. Another limitation is the use of only voting decision fusion, we plan to use other ensemble architectures, such as boosting and bagging. In addition, we will analyse the performance of multi-privacy protection scheme using score-fusion, taking into account protected user-specific scenarios.

As a future work we will focus in solving the proposal and scenario limitations, as well as, try to understand why scrolling scenarios shows worst results compared to horizontal strokes, to understand why BioConvolving has low EER values and to propose a new biometric protection method.

\section{REFERENCES}

[1] J. Feng and A. Jain, "Fingerprint reconstruction: From minutiae to phase," Pattern Analysis and Machine Intelligence, IEEE Transactions on, vol. 33, no. 2, pp. 209-223, Feb 2011.

[2] A. K. Jain, K. Nandakumar, and A. Nagar, "Biometric template security," EURASIP Journal on Advances in Signal Processing, vol. 2008, pp. 113:1-113:17, January 2008. [Online]. Available: http://dx.doi.org/10.1155/2008/579416

[3] N. Poh and S. Bengio, "How do correlation and variance of base-experts affect fusion in biometric authentication tasks?" Signal Processing, IEEE Transactions on, vol. 53, no. 11, pp. 4384-4396, 2005.

[4] A. K. Jain and A. Ross, "Multibiometric systems," Commun. ACM, vol. 47, no. 1, pp. 34-40, January 2004. [Online]. Available: http://doi.acm.org/10.1145/962081.962102

[5] N. Poh, A. Merati, and J. Kittler, "Heterogeneous information fusion: A novel fusion paradigm for biomet- ric systems," in Biometrics (IJCB), 2011 International Joint Conference on, Oct 2011, pp. 1-8.

[6] C. Rathgeb and C. Busch, "Multi-biometric template protection: Issues and challenges," in New Trends and Developments in Biometrics, D. J. Yang, Ed. INTECH Open Access Publisher, 2012. [Online]. Available: http://www.intechopen.com/books/new-trends-anddevelopments-in-biometrics/multi-biometric-templateprotection-issues-and-challenges

[7] M. Damasceno and A. Canuto, "An Empirical Analysis of Ensemble Systems in Cancellable Behavioural Biometrics: a Touch Screen Dataset," in 2014 International Joint Conference on Neural Networks (IJCNN 2014), IEEE. Beijing, China: IEEE, July 2014, in press.

[8] A. M. Canuto, F. Pintro, and J. ao C. Xavier-Junior, "Investigating fusion approaches in multi-biometric cancellable recognition," Expert Systems with Applications, vol. 40, no. 6, pp. 1971-1980, 2013.

[9] M. Damasceno and A. Canuto, "An Empirical Analysis of Ensemble Systems for Revocable Behavioural Biometric Verification," Journal of Information Assurance \& Security, vol. 9, no. 4, pp. 186-196, 2014.

[10] E. Kelkboom, X. Zhou, J. Breebaart, R. Veldhuis, and C. Busch, "Multi-algorithm fusion with template protection," in Biometrics: Theory, Applications, and Systems, 2009. BTAS'09. IEEE 3rd International Conference on. IEEE, 2009, pp. 1-8.

[11] L. I. Kuncheva, Combining Pattern Classifiers: Methods and Algorithms. John Wiley \& Sons, Inc, 2004.

[12] A. M. P. Canuto, M. C. Fairhurst, F. Pintro, J. C. X. Junior, A. F. Neto, and L. M. G. Gonalves, "Classifier ensembles and optimization techniques to improve the performance of cancellable fingerprint," International Journal of Hybrid Intelligent Systems, vol. 8, no. 3, pp. 143-154, 2011.

[13] A. B. Teoh, Y. W. Kuan, and S. Lee, "Cancellable biometrics and annotations on BioHash," Pattern Recognition, vol. 41, no. 6, pp. 2034-2044, 2008.

[14] E. Maiorana, M. Martinez-Diaz, P. Campisi, J. OrtegaGarcia, and A. Neri, "Template protection for hmmbased on-line signature authentication," in IEEE Conference on Computer Vision and Pattern Recognition Workshops (CVPRW), 2008, pp. 1-6.

[15] M. Frank, R. Biedert, E. Ma, I. Martinovic, and D. Song, "Touchalytics: On the Applicability of Touchscreen Input as a Behavioral Biometric for Continuous Authentication," in IEEE Transactions on Information Forensics and Security, vol. 8, no. 1, 2013, pp. 136-148. [Online]. Available: http://www.mariofrank.net/touchalytics/ 